with information about population structure from other such 'vagabond' fauna and flora, should continue to shed light on the vagaries of human evolution.

Christopher C. Austin

Evolutionary Biology Unit,

South Australian Museum,

Adelaide 5000, Australia

Present address: Institute of Statistical Mathematics, 4-6-7 Minami-Azabu Minato-ku,

Tokyo 106-8569, Japan

e-mail:caustin@ism.ac.jp

1. Diamond, J. M. Nature 336, 307-308 (1988).

2. Terrell, J. Antiquity 62, 642-657 (1988).

. Clegg, J. B. Lancet 344, 1070-1071 (1994).

4. Bellwood, P. S. in The Colonization of the Pacific: Some Current

Hypotheses (eds Hill, A. V. S. \& Serjeantson, S. W.) 1-59

(Oxford Univ. Press, 1989).

Kirch, P. V. \& Hunt, R. L. Radiocarbon 30, 161-169 (1988).

6. Pawley, A. \& Green, R. Ocean Ling. 12, 1-67 (1973).

7. Hagelberg, E. \& Clegg, J. B. Proc. R. Soc. Lond. B 252, 163-170 (1993)

8. Redd, A. J. et al. Mol. Biol. Evol. 12, 604-615 (1995).

9. Martinson, J. J. in Molecular Biology and Human Diversity (eds Boyce, A. J. \& Mascie-Taylor, C. G. N.) 171-195 (Cambridge Univ. Press, 1996).

10. Thorpe, R. S., McGregor, D. P., Cumming, A. M. \& Jordan, W. C. Evolution 48, 230-240 (1994).

\section{The first true inorganic fullerenes?}

Boron nitride and materials of composition $\mathrm{MX}_{2}$, where $\mathrm{M}$ is molybdenum or tungsten and $\mathrm{X}$ is sulphur or selenium, can form fullerene-like structures such as nested polyhedra or nanotubes ${ }^{1-3}$. However, the analogy to the carbon fullerene family ${ }^{4}$ falls short because no small preferred structure akin to $\mathrm{C}_{60}$ (ref. 5) has been found. We have discovered nano-octahedra of $\mathrm{MoS}_{2}$ of discrete sizes in soots that we prepared by laser ablation of pressed $\mathrm{MoS}_{2}$ targets. These nano-octahedra are much larger than $\mathrm{C}_{60}$ structures, having edge lengths of about 4.0 and 5.0 nanometres, and may represent the first 'inorganic fullerenes'.

Targets were prepared by pressing $98 \%$ pure $\mathrm{MoS}_{2}$ powder and ablated using a $\mathrm{KrF}$ pulsed excimer laser $(8 \mathrm{~Hz}, 248 \mathrm{~nm}, \sim 300$ mJ per pulse, $\sim 20 \mathrm{~ns}$ per pulse, $\sim 10 \mathrm{~J} \mathrm{~cm}^{-2}$ ) under flowing helium or argon (500-800 torr, $\left.\sim 90 \mathrm{~cm}^{3} \mathrm{~min}^{-1}\right)$. The beam was moved every 4 minutes during the 20 -minute runs to strike fresh target material, with the chamber and target temperature ranging from 30 to $600{ }^{\circ} \mathrm{C}$. The soot generated was collected, ultrasonicated in acetone, and applied to a grid for imaging by transmission electron microscopy (TEM).

Soot produced between 30 and $500{ }^{\circ} \mathrm{C}$ contained crystalline and amorphous $\mathrm{MoS}_{2}$ fractions, as well as smaller rhomboidal, rectangular and hexagonal structures 3 to 5 $\mathrm{nm}$ long with two or three layers. The crystalline material included large sheets and tubes and a variety of nested polyhedra 15 to $35 \mathrm{~nm}$ long that were similar to those produced previously ${ }^{6}$. Above $550{ }^{\circ} \mathrm{C}$, only crystalline folded sheets of $\mathrm{MoS}_{2}$ were produced.

TEM stage-tilting experiments on 30 two- and three-layered structures showed that the small rhomboids, rectangles and hexagons were different projections of the same three-dimensional structure: an octahedron (Fig. 1a). The TEM image for a closed three-layer structure changes with tilts of $10^{\circ}$ and $20^{\circ}$ (Fig. 1b). The image at $0^{\circ}$ is the projection expected for an octahedron orientated such that only two triangular faces are seen. When it is tilted, the projection changes, resulting in a nearly rectangular projection at $20^{\circ}$. Stick models depicting how an octahedron's projection changes with tilting are also shown in Fig. 1b. The model octahedron was orientated to project a match to the $0^{\circ}$ image, and the model was then tilted with the same axis used in the TEM. Many other TEM tilt


Figure 1 Structure of the molecules. a, Model octahedron with an edge length of 12 a lattice constants showing only the molybdenum sublattice. b, Transmission electron microscope images and modelgenerated projections for a three-layer $\mathrm{MoS}_{2}$ rhomboid at $0^{\circ}$ and undergoing tilts of $10^{\circ}$ and $20^{\circ}$. c, Histogram of octahedral edge lengths in a lattice constants determined from 30 rhomboidal, rectangular and hexagonal projections observed by TEM. sequences could also be generated with projections of a model octahedron. In some cases, slight movements of the particles on the TEM grid ruined the correlation, but individual images could still be represented by the projection of an octahedron.

The edge length of the octahedron may be calculated from TEM projections, assuming a regular octahedral structure. A histogram of edge lengths for 30 different structures is shown in Fig. 1c. Two pronounced peaks are seen at 12-13 and 16 times the $a$ lattice constant (the Mo-Mo distance, $3.16 \AA$ ) of $\mathrm{MoS}_{2}$ for two- and three-layer species, respectively. The spacing between the layers is about $0.6 \mathrm{~nm}$, in good agreement with the interlayer spacing in bulk $\mathrm{MoS}_{2}$. The edge of the three-layer species is about four $a$ lattice constants larger than that of the underlying two-layer structure, exactly the size required to maintain the bulk interlayer spacing.

Although the reasons for these specific sizes are not clear, a preference for two- and three-layer structures may be associated with the two- and three-layer polytypes ${ }^{7}$. The octahedral shape might be anticipated for a closed $\mathrm{MoS}_{2}$ structure as the triangular faces share the symmetry of the trigonal Mo and $\mathrm{S}$ sublattices. Rounded corners and edges are also expected for $\mathrm{MoS}_{2}$ sheets, which cannot be severely bent without strain. Energy-dispersive spectroscopy indicated a Mo:S ratio of about 1:2 with no detectable impurities. Satisfying such a ratio exactly is impossible in an octahedron, but several arrangements come close. For example, the Mo-S coordination could remain trigonal prismatic, as in the bulk form, with a given face being slightly rich or poor in sulphur. The structure at the vertices is unclear, but either a four-membered Mo ring $^{3}$ or a single Mo atom might be stable (B. Parkinson, personal communication).

TEM measurements could be performed only on nano-octahedra that were separated from the agglomerates formed on the TEM grid. Consequently, we cannot yet estimate the density of nano-octahedra in the laser-generated soots. We are purifying these inorganic fullerenes so that we can ascertain their properties, and are also finding out whether similar cage structures can be made using other layered materials.

P. A. Parilla, A. C. Dillon, K. M. Jones, G. Riker, D. L. Schulz, D. S. Ginley,

\section{J. Heben}

National Renewable Energy Laboratory, 1617 Cole Boulevard, Golden,

Colorado 80401-3393, USA

\footnotetext{
1. Tenne, R., Margulis, L., Genut, M. \& Hodes, G. Nature 360, 444-446 (1992).

2. Chopra, N. G. et al. Science 269, 966-967 (1995).

3. Tenne, R. Adv. Mater. 7, 965-995 (1995).

4. Kroto, H. Science 242, 1139-1145 (1988).

5. Kroto, H. W. et al. Nature 318, 162-163 (1985).

6. Feldman, Y., Wasserman, E., Srolovitz, D. J. \& Tenne, R. Science 267, 222-225 (1995)

7. Wilson, J. A. \& Yoffe, A. D. Adv. Phys. 18, 193-335 (1969).
} 